\title{
Comparative assessment of antibacterial efficacy for cobalt nanoparticles, bulk cobalt and standard antibiotics: A concentration dependant study
}

\author{
V. Gupta ${ }^{1 *}$, V. Kant ${ }^{2}$, A. K. Sharma ${ }^{1}$, M. Sharma ${ }^{1}$ \\ ${ }^{1}$ Department of Chemistry, University of Jammu, J\&K, India \\ ${ }^{2}$ Department of Veterinary Pharmacology \& Toxicology, LUVAS, Hisar, India \\ *vijayta1gupta@gmail.com
}

DOI 10.17586/2220-8054-2020-11-1-78-85

\begin{abstract}
Synthesis of compounds that can prevent bacterial resistance is of huge interest and gaining immense popularity. Cobalt (Co) is one of the cheaper transition metals and its nano form has not been studied in details for antibacterial actions. Comparative analysis of Co nanoparticles with bulk Co and standard antibacterials are also lacking. In our study, concentration dependent action of Co nanoparticles was observed from 0.125 to $128.0 \mu \mathrm{g} / \mathrm{ml}$ against $S$. aureus and E. coli. Zone of inhibition of Co nanoparticles was better against E. coli than S. aureus. Co nanoparticles were markedly betterthan bulk Co, oxytetracycline and gentamicin. Activity index and fold increase of Co nanoparticles were higher at most of the concentrations. In conclusion, Co nanoparticles showed better antibacterial action than other tested compounds against $S$. aureus and E. coli particularly at lower concentrations, and their use may be extended in different biomedical fields in future.
\end{abstract}

Keywords: Cobalt nanoparticles, S. aureus, E. coli, antibacterial activity, activity index, fold increase.

Received: 6 November 2019

Revised: 10 December 2019

\section{Introduction}

There is a continuous battle throughout history between humans and multiple microorganisms. Major discoveries and developmentsfor antibacterial drugs and other ways of infection control during the middle of the 20th century have occurred, which helped significantly for human being and animals. However, rapid emergence of bacterial resistance become as one of the major concerns of the 21st century. Antimicrobial resistance has become a global problem with the fast increase in multidrug-resistant bacteria. Staphylococcus aureus (S. aureus) and Escherichia coli (E. coli) are two common bacteria to which almost every human being and animal has been exposed. Some earlier treatable microorganisms are currently becoming untreatable such as methicillin-resistant Staphylococcus aureus (MRSA) and vancomycin-resistant enterococcus (VRE) [1]. Different strategies to address this challenge include the designing of improved version of existing antibacterial classes, combinations therapy etc. have been attempted. These strategies can be quite effective, but a high risk of rapid development of bacterial resistance still remains. Bacteria have the genetic capability to transmit and acquire resistance to antimicrobial drugs. In the last three decades, different pharmaceutical industries have produced a lot of new antibiotics, but the bacterial resistance to these drugs has also increased [2]. In view of the increase of bacterial resistance, synthesis of compounds that may prevent bacterial growth with least cytotoxicity are of huge interest and gaining immense popularity in the area of drug development.

During past decades, applications of nanotechnology have brought many breakthroughs. In recent years, much attention has been given to the applications of nanotechnology in various metals and metal oxides for their different uses. Metal nanoparticles are exceptionally appealing better applications in contrast to mass materials due to their high surface-to-volume proportion. There are many nano metals (such as silver, gold, copper, zinc etc.), which exhibit their own structural properties and important biological activities [3,4]. Metals have been used in the therapy of several diseases of humans and animals since ancient times. Copper and cobalt (Co) ions have been used for centuries by people to inhibit the growth of harmful microbes. Further, transition metals and their complexes are of current interest from numerous point of view, like their use as antioxidant, antibacterial, antifungal, anticancer, anti-inflammatory agent etc. $[5,6]$.

Co is one of the cheaper transition metals which have wide applications and also possesses antimicrobial properties. Co nanoparticles are of significant interests due to their exceptional magnetic and catalytic properties, and can be used in super alloys, magnetic fluids, computer hard disks, magnetic sensor, catalyst etc [7]. Moreover, Co nanoparticles are also more focused in healthcare system now days due to its antiseptic action. The concentration dependent studies demonstrating antibacterial potentials of Co nanoparticles are very limited, and the studies on concentration dependent comparative efficacy for antibacterial actions of Co nanoparticles with respect to its bulk form as well as standard antibiotics are completely lacking to the best of our knowledge. In this context, we focused the attention on the synthesis of Co nanoparticles to determine their antibacterial efficacy against $S$. aureus and E. coli at different 
concentrations, and their comparative analysis of antibacterial potentials with respect to bulk Co, oxytetracycline and gentamicin at different concentrations.

\section{Materials and methods}

\subsection{Materials used}

The different chemicals of analytical grade were used for this investigation. Cobalt chloride hexahydrate $\left(\mathrm{CoCl}_{2}\right.$. $6 \mathrm{H}_{2} \mathrm{O}$ ), hydrazine hydrate, sodium hydroxide and ethylene glycol were used for the synthesis of Co nanoparticles/nanofluids and purchased from Sigma Aldrich, USA. Dimethyl sulfoxide (DMSO) was purchased from SRL. Bulk Co powder was purchased from Central Drug House (CDH), New Delhi, India. Oxytetracycline and gentamicin were procured from Hi-media, Mumbai. The Muller-Hinton Agar (MHA), Muller-Hinton Broth (MHB) and nutrient broth (NB) were purchased from Hi-media, Mumbai, India and used for antibacterial studies. The S. aureus (MTCC 1430) and E. coli (MTCC 2127) were two bacterial strains in used in this study. These were gifted from the Department of Biotechnology, University of Jammu and revived by using the nutrient broth (NB).

\subsection{Synthesis and characterization of Co nanoparticles}

The synthesis of cobalt nanoparticles was carried out by reducing a cobalt(II) salt using hydrazine hydrate as reducing agent according to the method of Zhu et al. [8] with some modifications. In a typical procedure, cobalt chloride (10 g) was dissolved into $150 \mathrm{ml}$ ethylene glycol and water mixture (2:1). The solution was stirred vigorously until it completely dissolves. The $\mathrm{pH}$ of the solution was adjusted to 12 using aqueous $\mathrm{NaOH}$ solution. The solution of $\mathrm{CoCl}_{2}$ was then treated with the mixture of hydrazine hydrate $(50 \%)$. After about 30 min, black solid particles appeared. After the completion of reaction, the reaction mixture was centrifuged. The black particles collected were washed several times with triply distilled water and absolute ethanol to remove hydrazine, sodium, and chlorine ions. The final product was then dried in an oven at $60{ }^{\circ} \mathrm{C}$.

The reduction reaction can be expressed as:

$$
2 \mathrm{Co}^{2+}+\mathrm{N}_{2} \mathrm{H}_{4}+4 \mathrm{OH}^{-} \rightarrow 2 \mathrm{Co}+\mathrm{N}_{2}+4 \mathrm{H}_{2} \mathrm{O}
$$

The characterization studies of synthesized nanoparticles were conducted by using various standard techniques like particle size analyzer (PSA), transmission electron microscopy (TEM) and scanning electron microscopy (SEM). The particle size distribution (PSD) study of the synthesized nanoparticles was conducted by using Malvern Instruments Zetasizer Nano-ZS instrument. The evaluation of the size and morphology of Co nanoparticles was done by using transmission electron microscope (Hitachi H-7500). The scanning electron microscope (SEM-EDS) using SEM (JEOL JSM - 6390LV) was used for the analysis of surface morphology of the Co nanoparticles.

\subsection{Assessment of antibacterial efficacy}

All the antimicrobial activity assays were performed in triplicate and confirmed by three independent experiments. The antimicrobial activity of synthesized Co nanoparticles and its comparison with oxytetracycline, gentamicin and bulk Co was investigated against the gram-positive i.e. S. aureus and gram-negative i.e. E. coli bacterial strains. The in vitro antibacterial activity was conducted as per agar well diffusion method by using Muller-Hinton agar.The overnight bacterial cultures $\left(108 \mathrm{cfu} / \mathrm{ml}\right.$ ) were used for seeding of the autoclaved (at $121{ }^{\circ} \mathrm{C}$ and $15 \mathrm{lbs}$ ) Mueller Hinton agar medium. Seeded agar was later on poured in glass petri plates $(30 \mathrm{ml})$ and allowed for its solidification. Six wells of $6 \mathrm{~mm}$ diameter were created into the agar plates and sealed with one drop of $0.8 \%$ melted agar to prevent the leakage of tested compounds. Oxytetracycline, gentamicin, bulk Co and Co nanoparticles were poured (30 $\mu \mathrm{l}$ of each dilution) in the different wells and incubated at $37{ }^{\circ} \mathrm{C}$ for $24 \mathrm{hrs}$. The sensitivities of the bacterial strains to the different concentrations of tested compounds were indicated by clear zone around the wells. Diameters of zone of inhibition produced by of different concentrations of tested compounds were measured to quantify the antibacterial potentials. In present study, DMSO was used as negative control (NC), and oxytetracycline and gentamicin were used as positive control to compare the efficacy of the nanoparticles. Activity index (AI) and fold increase for the comparison of antibacterial potentials of Co nanoparticles with bulk Co, oxytetracycline and gentamicin was also calculated by using the values of zone of inhibition.

$$
\begin{gathered}
\text { Activity Index }(\mathrm{AI})=\frac{\text { Inhibition zone of the Co nanoparticles }}{\text { Inhibition zone of the bulk Co or oxytetracycline or gentamicin }}, \\
\text { Fold increase }(\%)=\left(\frac{b-a}{a}\right) 100,
\end{gathered}
$$

where, $a$ and $b$ refer to the inhibition zones of oxytetracycline/gentamicin/bulk Co and Co nanoparticles, respectively. 


\section{Results and discussion}

Figure 1A is representing the PSD of Co nanoparticles. Sample of Co nanoparticles gives an overall z-average size of $145.6 \mathrm{~nm}$ with polydispersity index of 0.372 , which revealed that the distribution consists of a single size mode without aggregates. TEM (Fig. 1B) and SEM (Fig. 1C) micrographs of Co nanoparticles showed that synthesized Co nanoparticles have spherical morphology and average size of $13-25 \mathrm{~nm}$. SEM image also indicated that the prepared nanoparticles are of spherical shape. SEM picture specifies that individual nanoparticles are aggregated and give large sphere like cumulative structure.

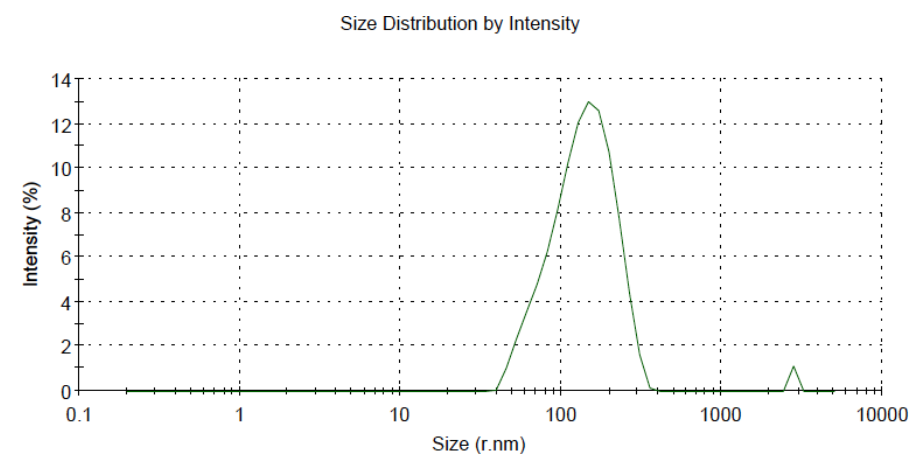

(A)

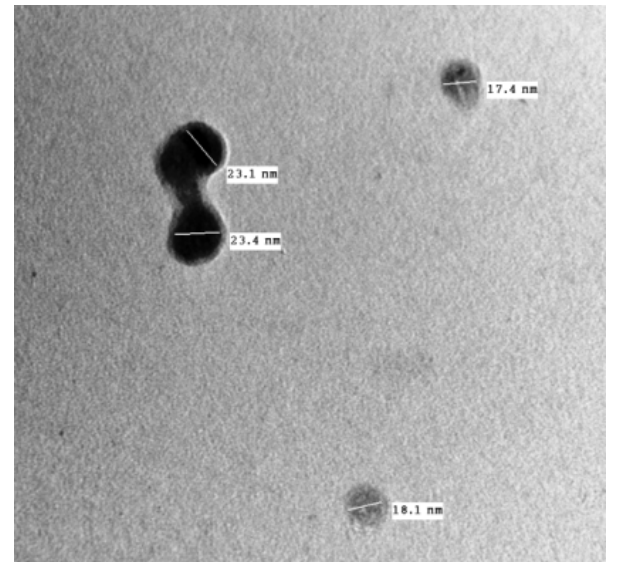

(B)

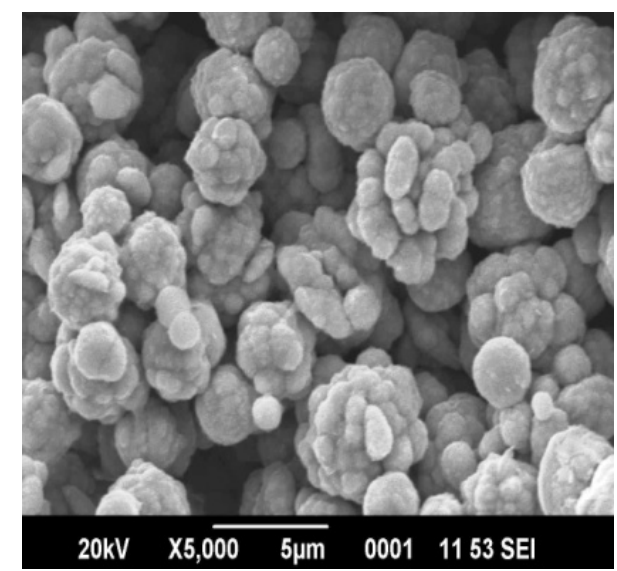

(C)

FIG. 1. (A) The particle size distribution (PSD) of Co nanoparticles. Micrograph of TEM (B) and SEM (C) of Co nanoparticles

In this study the synthesis of cobalt nanoparticles was followed as per the Zhu et al. method [8] with some modifications. But, the size of Co nanoparticles in our study was very smaller than the size observed in the study of Zhu et al. [8]i.e. 1 to $2 \mu \mathrm{m}$. This variation in size in our and study of Zhu et al. [8] was might be due to the variations in the reaction media. The pure ethylene glycol was used as solvent in the study of Zhu et al. [8], where as ethylene glycol and water mixture $(2: 1)$ was used in our study. Previous studies have also reported that solvents usually influence the growth and the morphologies of crystals, and even the formation and the structure of crystals [9-11], because the solvents can selectively control the surface energy of different crystallographic faces $[12,13]$.

Figure 2 is showing the representative images of bacterial culture plates with zone of inhibition produced by different concentrations ofoxytetracycline, gentamicin, bulk Co and Co nanoparticles against S. aureus and E. coli. Table 1 shows the mean value of diameters of zone of inhibition for oxytetracycline, gentamicin, bulk Co and Co nanoparticles at different concentrations against both bacteria. In many of the earlier studies, both $S$. aureus and E. coli are routinely used to evaluate the antimicrobial activities of diverse nanoparticles [14, 15]. We also selected these two bacterial strains and found that synthesized Co nanoparticles produced larger zone of inhibition against $E$. coli than $S$. aureus at the same concentration. This indicated that action of Co nanoparticles is stronger against $E$. coli than S. aureus. 

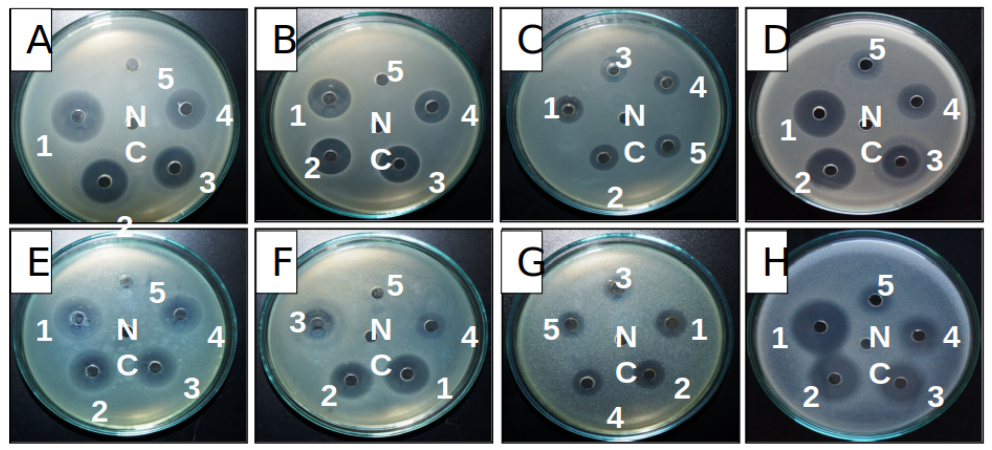

FIG. 2. Zone of inhibitions of oxytetracycline (A \& E), gentamicin (B \& F), bulk Co (C \& G) and Co nanoparticles (D \& H) against S. aureus (A-D) and E. coli (E-H) at different concentrations i.e. (1) $128.0 \mu \mathrm{g} / \mathrm{ml}$, (2) $32.0 \mu \mathrm{g} / \mathrm{ml}$, (3) $8.0 \mu \mathrm{g} / \mathrm{ml}$, (4) $2.0 \mu \mathrm{g} / \mathrm{ml}$, and (5) $0.5 \mu \mathrm{g} / \mathrm{ml}$. NC represents negative control i.e. DMSO

TABLE 1. Zone of inhibition for oxytetracycline, gentamicin, bulk Co and Co nanoparticles (NPs) against $S$. aureus and E. coli at different concentrations

\begin{tabular}{|c|c|c|c|c|c|c|c|c|}
\hline \multirow{2}{*}{$\begin{array}{c}\text { Conc. } \\
(\boldsymbol{\mu g} / \mathbf{m l})\end{array}$} & \multicolumn{3}{|c|}{ Zone of inhibition (mm) against $\boldsymbol{S}$ a aureus } & \multicolumn{3}{c|}{ Zone of inhibition (mm) against $\boldsymbol{E}$. coli } \\
\cline { 2 - 10 } & Oxytetracycline & Gentamicin & Bulk Co & Co NPs & Oxytetracycline & Gentamicin & Bulk Co & Co NPs \\
\hline 128.0 & 20.92 & 17.33 & 9.50 & 20.17 & 19.33 & 16.83 & 11.33 & 21.50 \\
\hline 64.0 & 19.17 & 14.17 & 9.33 & 18.00 & 18.50 & 13.83 & 10.67 & 19.33 \\
\hline 32.0 & 17.17 & 13.17 & 9.25 & 17.00 & 16.67 & 12.67 & 9.83 & 18.17 \\
\hline 16.0 & 16.17 & 11.83 & 9.00 & 15.83 & 15.33 & 11.33 & 9.50 & 17.00 \\
\hline 8.0 & 15.42 & 10.92 & 8.92 & 15.33 & 14.42 & 10.33 & 9.33 & 15.75 \\
\hline 4.0 & 14.17 & 10.58 & 8.67 & 13.67 & 12.83 & 9.17 & 9.25 & 15.00 \\
\hline 2.0 & 13.00 & 10.08 & 8.50 & 12.83 & 12.17 & 8.17 & 9.00 & 14.17 \\
\hline 1.0 & 8.50 & 8.17 & 8.33 & 11.75 & 8.17 & 7.17 & 8.92 & 13.33 \\
\hline 0.5 & 0.00 & 0.00 & 8.08 & 10.67 & 0.00 & 0.00 & 8.67 & 11.92 \\
\hline 0.25 & 0.00 & 0.00 & 7.92 & 9.33 & 0.00 & 0.00 & 8.50 & 11.17 \\
\hline 0.125 & 0.00 & 0.00 & 7.50 & 8.50 & 0.00 & 0.00 & 8.25 & 9.67 \\
\hline
\end{tabular}

Growth and survival of bacteria is also affected by size, morphology and concentration of nanoparticles. Previous studies have suggested that metal and metal oxide nanoparticles having 1 - $100 \mathrm{~nm}$ size and different shapes might have promising antimicrobial actions [16]. Thus, size of $13-25 \mathrm{~nm}$ and spherical shape of our synthesized Co nanoparticles revealed that our Co nanoparticles might be considered a promising antimicrobial candidate. The smaller size of the metal nanoparticles than the pores of bacterial cell membrane helps the easy transmission of nanoparticles across the cell wall to the cytoplasm, which further causes cell death [17]. Thus, the potent antibacterial potentials of Co nanoparticles might be attributed to their relatively smaller sizes and high amount of surface-area-to-volume ratio which facilitated close interaction with the bacterial membranes [18]. Earlier, it has been also reported that inhibition of bacterial growth increases with the increase in concentration of nanoparticles [19]. In our study, concentration dependent increase in diameter of zone of inhibition for Co nanoparticles was observed only up to $128.0 \mu \mathrm{g} / \mathrm{ml}$ against both bacteria, and beyond this concentration, the size of the zone started to decrease. This might be due to the agglomeration of nanoparticles beyond these concentrations. Previously, it has been reported that optimum concentration exist for nanoparticles at which higher effects are noticed in comparison to more or less concentration [20]. Thus, in 
this study, $128.0 \mu \mathrm{g} / \mathrm{ml}$ was the optimum concentrations for Co nanoparticles against $S$. aureus and E. coli. Oxytetracycline, gentamicin and bulk Co showed increases in diameters of zones of inhibition in concentration dependent manners against both bacteria at all the tested concentrations. Oxytetracycline and gentamicin did not show zone of inhibitions at lower concentrations, i.e., below $1.0 \mu \mathrm{g} / \mathrm{ml}$ against $S$. aureus and E. coli (Table 1). Thus, oxytetracycline and gentamicin were considered ineffective at lower concentrations. However, synthesized Co nanoparticles of present study were effective below these concentrations and produced marked zone of inhibition up to $0.125 \mu \mathrm{g} / \mathrm{ml}$. The bulk Co also showed some antibacterial activity at all the tested concentrations, but the sizes of the zones of inhibition produced by it were of smaller size in comparison to it nano form. These results suggested that the range of inhibitory concentration for nano Co was broader with respect to oxytetracycline and gentamicin. Nano form of Co possesses much better antibacterial efficacy in comparison to its bulk form. The markedly better antibacterial effects of nano form of Co particularly at lower concentrations than other tested compounds of present study also revealed that Co nanoparticles may provide antibacterial action for longer duration with less frequency of applications. In the coming years, Co nanoparticles may be considered as a substitute for oxytetracycline, gentamicin and bulk Co in different fields in relation to reduce the occurrence of antibacterial resistance.

Earlier studies have also shown the antibacterial actions of Co nanoparticles prepared by different methods. Environmentally-benign synthesis of Co nanoparticles by using secondary metabolites from Celosia argentea plant extract have showed antibacterial activity against E. coli and Bacillus subtilis (B. subtilis) [21]. Studies have revealed that Co nanoparticles possesses potential for the development of antiamoebic nanomedicine. In a recent study, different varieties of Co nanoparticles synthesized by hydrothermal and ultrasonication methods have been found effective against amoeba species like Acanthamoeba castellanii (causative agent for threatening Acanthamoeba keratitis and granulomatous amoebic encephalitis) [22]. The results of this study revealed that cobalt phosphate $\mathrm{Co}_{3}\left(\mathrm{PO}_{4}\right)_{2}$ hexagonal microflakes, and $100 \mathrm{~nm}$ large cobalt hydroxide $\left(\mathrm{Co}(\mathrm{OH})_{2}\right)$ nanoflakes showed potent amoebicidal activity as compared to granular cobalt oxide $\left(\mathrm{Co}_{3} \mathrm{O}_{4}\right)$. Further, other types of Co based nonmaterials have also shown the antimicrobial actions. Cobalt oxide $\left(\mathrm{Co}_{2} \mathrm{O}_{4}\right)$ nanoparticles have revealed the concentration $(1,50$ and $100 \mathrm{mg} / \mathrm{ml}) \mathrm{de}-$ pendent antibacterial activity against different bacterial strains like E.coli, Pseudomonas aeruginosa (P. aeruginosa) and $B$. subtilis [23]. Cobalt ferrite $\left(\mathrm{CoFe}_{2} \mathrm{O}_{4}\right)$ nanoparticles have shown antibacterial activity against $E$. coli and its minimum inhibitory concentration was found at $50 \mathrm{mg} / \mathrm{L}$ [24]. A comparative study of cobalt ferrite $\left(\mathrm{CoFe}_{2} \mathrm{O}_{4}\right)$ and silver-cobalt ferrite $\left(\mathrm{Ag}-\mathrm{CoFe}_{2} \mathrm{O}_{4}\right)$ nanoparticles, synthesized by wet ferritization and self-combustion methods using Hibiscus rosa-sinensis flower/leaf extracts, showed inhibitory actions against the different strains of bacteria and fungus, and the $\mathrm{Ag}-\mathrm{CoFe}_{2} \mathrm{O}_{4}$ nanoparticles revealed better inhibitory potentials than $\mathrm{CoFe}_{2} \mathrm{O}_{4}$ nanoparticles obtained by either of the two methods [25].

The surface of the nanoparticles has a significant effect in their bioactivity and materials made up of nanoparticles have a much greater surface area per unit volume as compared to the materials made up of bigger particles (bulk materials). This makes nanoparticles more reactive and interactive to cell wall/membrane, which leads to their impairment and results in leakage of cellular contents [26]. Previous study has revealed that the antibacterial activity of the nanomaterials increased with an increase in the surface-to-volume ratio due to the decrease in size of nanoparticles, and the size of the nanoparticles might play a role in the antibacterial activity [17]. Although, the exact mechanisms by which the metallic nanoparticles produce antibacterial actions are not clear and still under debate. Some possible mechanisms are supposed by which they produce actions include, interaction of nanoparticles with the cell wall/membrane, formation of reactive oxygen species (ROS), release of metal ions etc. Electrostatic attraction, vander waals forces, receptor - ligand interactions and hydrophobic interactions are considered different ways which promote nanoparticles and bacterial contact [27-30]. Nanoparticles can cross microbe membranes after making contact and interfere with different bacterial metabolic pathways, and also induce changes in membrane shape and permeability. Nanoparticles inside cells interact with the different microbial components and cause inhibition of enzymes, deactivation of proteins, production of oxidative stress, modulate the gene expression and electrolyte imbalance [31]. Previous studies have revealed that different metal nanoparticles produce various types of ROS, which resulted in their antibacterial actions [32]. Different types of ROS are observed to inhibit transcription, translation, enzymatic activity, and the electron transport chain in bacteria [33,34]. Metal ions also have tendency to attach and destroythe DNA molecule after entry into the bacterial cell [26]. It has been observed that metallic nanoparticles have enhanced potential to combat bacterial infections as compared to their salts, and the size of nanoparticles mostly influences the antibacterial mechanism [33,35-37]. The fact that small sized nanoparticles tend to be more toxic to bacteria than large nanoparticles and their bulk form is due to the larger surface area to volume ratio of small nanoparticles [38]. So, the antibacterial actions by Co metal nanoparticles in this study might be due through different ways, which could be the reason for its better effects as compared to standard antimicrobials.

The calculated values of activity index (AI) as well as fold increase (\%) are used to analyze the comparative antimicrobial efficacy of testedcompound with respect to other compound [39]. The tested drug/compound can be 
considered better against a particular bacterial strain, if its AI value is more than one. In our study, the AI for Co nanoparticles with respect to (w.r.t.) oxytetracycline, gentamicin and bulk Co against $S$. aureus and E. coli were calculated at various concentrations, and the values are presented in Table 2. The AI of Co nanoparticles was higher than 1 w.r.t. oxytetracycline upto $1.0 \mu \mathrm{g} / \mathrm{ml}$ against $S$. aureus and at all the tested concentrations against $E$. coli. The AI of Co nanoparticles w.r.t. gentamicin and bulk Co was higher than 1 at all the tested concentrations against both bacterial strains. The calculation of fold increase (\%) is another approach to determine the relative effect of the tested compound in contrast to other compounds. The positive value of fold increase reveals the better potentials of tested compound. However, negative value of fold increase reveals lesser efficacy of the tested compound than other compound against a particular bacterial strain at the same concentration. The Tables 3 presents the values of fold increase for Co nanoparticles w.r.t. oxytetracycline, gentamicin and bulk Co against S. aureus and E. coli at different concentrations. We observed in this study that the positive values of fold increase of Co nanoparticles w.r.t. other tested compounds against both bacterial strains supported the observations of AI. These findings are suggesting that Co nanoparticles may have better antibacterial potentials, particularly at lower concentrations, than other tested compounds of present study. So, Co nanoparticles may be used for applications/coating of different larger surface area, cellulose bandages, uniforms, bed linen, medical equipment, different utensils of medical fields etc., which may be useful as preventive approach to control microbial infections. This will make a substantial impact to reduce the hospitalized bacterial contamination, mortality, costs of treatment etc.

TABLE 2. Activity index of Co nanoparticles with respect to (w.r.t.) oxytetracycline, gentamicin and bulk Co against $S$. aureus and $E$. coli at different concentrations

\begin{tabular}{|c|c|c|c|c|c|c|}
\hline \multirow{2}{*}{$\begin{array}{c}\text { Conc. } \\
(\boldsymbol{\mu g} / \mathbf{m l})\end{array}$} & \multicolumn{2}{|c|}{$\begin{array}{c}\text { Activity Index } \\
\text { w.r.t. Oxyttracycline }\end{array}$} & \multicolumn{2}{c|}{$\begin{array}{c}\text { Activity Index } \\
\text { w.r.t. Gentamicin }\end{array}$} & \multicolumn{2}{c|}{$\begin{array}{c}\text { Activity Index } \\
\text { w.r.t. bulk Co }\end{array}$} \\
\cline { 2 - 7 } & S. aureus & E. coli & S. aureus & E. coli & S. aureus & E. coli \\
\hline 128.0 & 0.96 & 1.11 & 1.16 & 1.28 & 2.12 & 1.90 \\
\hline 64.0 & 0.94 & 1.05 & 1.27 & 1.40 & 1.93 & 1.81 \\
\hline 32.0 & 0.99 & 1.09 & 1.29 & 1.43 & 1.84 & 1.85 \\
\hline 16.0 & 0.98 & 1.11 & 1.34 & 1.50 & 1.76 & 1.79 \\
\hline 8.0 & 0.99 & 1.09 & 1.40 & 1.52 & 1.72 & 1.69 \\
\hline 4.0 & 0.96 & 1.17 & 1.29 & 1.64 & 1.58 & 1.62 \\
\hline 2.0 & 0.99 & 1.16 & 1.27 & 1.73 & 1.51 & 1.57 \\
\hline 1.0 & 1.38 & 1.63 & 1.44 & 1.86 & 1.41 & 1.50 \\
\hline 0.5 & - & - & - & - & 1.32 & 1.38 \\
\hline 0.25 & - & - & - & - & 1.18 & 1.31 \\
\hline 0.125 & - & - & - & - & 1.13 & 1.17 \\
\hline
\end{tabular}

Studies of last few years have showed that the applications of nanoforms of various metals like $\mathrm{Ag}, \mathrm{Cu}, \mathrm{Tior} \mathrm{Zn}$ etc has been augmented due to their bactericidal action against different bacterial strains. Nanoparticles of $\mathrm{Ag}, \mathrm{Cu}$, $\mathrm{Zn}$ etc. are commonly and widely employed for different purposes like bactericides in catheters, burn wound care, dental practices, antibacterial soaps etc. [40]. However, use of Ag nanoparticles has started to decline due to biological safety of Ag nanoparticles, pigmentation effect of Ag nanoparticles on teeth etc [41]. The Co, metal used in this study, is considered cheaper than silver. In the future, Co nanoparticles may replace the silver nanoparticles for various applications. Additionally, metal nanoparticles are considered better antimicrobial pharmaceuticals in comparison to organic nanoparticles due to their durability, high stability, lower mammalian cell toxicity etc. [42]. The promising antimicrobial efficacy revealed by Co nanoparticles might be very useful for several therapeutic applications in biomedical sciences. Applications of Co nanoparticles may also be extended to food industry, textile industry, nursing homes, water purification, air purifications, paint industry, sewage treatment, etc. There may also be new construction of numerous materials such as antibacterial plastics, antibacterial ceramics, antibacterial clothes, antibacterial stainless steels etc. by using Co nanoparticles. Moreover, applications of Co nanoparticles coated materials in hospitals 
TABLE 3. Fold increase (\%) of Co nanoparticles with respect to (w.r.t.) oxytetracycline, gentamicin and bulk Co against $S$. aureus and $E$. coli at different concentrations

\begin{tabular}{|c|c|c|c|c|c|c|}
\hline \multirow{2}{*}{$\begin{array}{c}\text { Conc. } \\
(\boldsymbol{\mu g} / \mathbf{m l})\end{array}$} & \multicolumn{2}{|c|}{$\begin{array}{c}\text { Fold increase (\%) } \\
\text { w.r.t. Oxytetracycline }\end{array}$} & \multicolumn{2}{c|}{$\begin{array}{c}\text { Fold increase (\%) } \\
\text { w.r.t. Gentamicin }\end{array}$} & \multicolumn{2}{c|}{$\begin{array}{c}\text { Fold increase (\%) } \\
\text { w.r.t. bulk Co }\end{array}$} \\
\cline { 2 - 7 } & S. aureus & E. coli & S. aureus & E. coli & S. aureus & E. coli \\
\hline 128.0 & -3.59 & 11.21 & 16.35 & 27.72 & 112.28 & 89.71 \\
\hline 64.0 & -6.09 & 4.50 & 27.06 & 39.76 & 92.86 & 81.25 \\
\hline 32.0 & -0.97 & 9.00 & 29.11 & 43.42 & 83.78 & 84.75 \\
\hline 16.0 & -2.06 & 10.87 & 33.80 & 50.00 & 75.93 & 78.95 \\
\hline 8.0 & -0.54 & 9.25 & 40.46 & 52.42 & 71.96 & 68.75 \\
\hline 4.0 & -3.53 & 16.88 & 29.13 & 63.64 & 57.69 & 62.16 \\
\hline 2.0 & -1.28 & 16.44 & 27.27 & 73.47 & 50.98 & 57.41 \\
\hline 1.0 & 38.24 & 63.27 & 43.88 & 86.05 & 41.00 & 49.53 \\
\hline 0.5 & - & - & - & - & 31.96 & 37.50 \\
\hline 0.25 & - & - & - & - & 17.89 & 31.37 \\
\hline 0.125 & - & - & - & - & 13.33 & 17.17 \\
\hline
\end{tabular}

may help reduce the prevalence of nosocomial infections. The utilization of Co nanoparticles coated materials in many industries may also be beneficial to check bacterial deterioration of material. Combination therapy of metal nanoparticles with antibiotics has showed some synergistic effects $[43,44]$. Therefore, use of combination therapy of Co nanoparticles with standard antibiotics against simple or mixed type microbial infections may be extended for the formulations of more efficacious preparations.

\section{Conclusion}

In conclusion, the Co nanoparticles possess much better antibacterial efficacy than its bulk form and are also better than standard antibacterials, particularly at lower concentrations, against $S$. aureus and E. coli. In future, the strong antibacterial potentials of Co nanoparticles may extend its applications in different fields and could create a great revolution in the field of health care.

\section{Acknowledgements}

We are thankful to Department of Chemistry and Department of Biotechnology, University of Jammu(J\&K), for providing essential facilities and support for conducting present investigation. The first author is highly thankful to CSIR UGC, New Delhi, India for providing the Junior Research Fellowship (JRF). The authors also acknowledge the support of SAIF STIC (Kochi), SAIF Chandigarh, CIL Chandigarh for providing the facilities for characterization of nanoparticles.

\section{References}

[1] Henderson D.K. Managing methicillin-resistant staphylococci: a paradigm for preventing nosocomial transmission of resistant organisms. Am. J. Infect. Control, 2006, 34, P. S46-S54, S64-S73.

[2] Faúndez G., Troncoso M., Navarrete P., Figueroa G. Antimicrobial activity of copper surfaces against suspensions of Salmonella enterica and Campylobacter jejuni. BMC Microbiol., 2004, 4, P. 19-26.

[3] Siddiqi K.S., Rahman A., Tajuddin, Husen A. Properties of Zinc Oxide Nanoparticles and Their Activity against Microbes. Nanoscale Res. Lett., 2018, 13, P. 2-13.

[4] Peterson E., Kaur P. Antibiotic Resistance Mechanisms in Bacteria: Relationships between Resistance Determinants of Antibiotic Producers, Environmental Bacteria, and Clinical Pathogens. Front. Microbiol., 2018, 9, P. 2-21.

[5] Ghosh A.K., Mitra M., et al. Antibacterial and catecholase activities of Co (III) and Ni (II) Schiff base complexes. Polyhedron, 2016,107, P. 1.

[6] Mjos K.D., Orvig C. Metallodrugs in medicinal inorganic chemistry. Chem. Rev., 2014, 114 (8), P. 4540-4563.

[7] Zhang L., Lan T., et al. Template-free Synthesis of One-dimensional Cobalt Nanostructures by Hydrazine Reduction Route. Nanoscale Res. Lett., 2011, 6, P. 58. 
[8] Zhu Y., Zheng H., et al. Growth of Dendritic Cobalt Nanocrystals at Room Temperature. J. Cryst. Growth, 2004, 260, P. 427-434.

[9] Yang Q., Tang K., et al. PVA-Assisted Synthesis and Characterization of CdSe and CdTe Nanowires. J. Phys. Chem. B, 2002, 106, P. 92279230.

[10] Yang Q., Tang K., et al. Wet Synthesis and Characterization of MSe (M = Cd, Hg) Nanocrystallites at Room Temperature. J. Mater. Res., 2002, 17 (5), P. 1147-1152.

[11] Yang Q., Wang F., et al. The formation of fractal Ag nanocrystallites via $\gamma$-irradiation route in isopropyl alcohol. J. Mater. Chem. Phys., 2002, 78, P. 495-500.

[12] Lee S.M., Cho S.N., Cheon J. Anisotropic Shape Control of Colloidal Inorganic Nanocrystals. Adv. Mater., 2003,15 (5), P. $441-444$.

[13] Puntes V.F., Kishnan K.M., Alivisatos A.P. Colloidal nanocrystal shape and size control: the case of cobalt. Science, 2001, 291 (5511) P. 2115-2117.

[14] Yoon K.Y., Byeon J.H., Park J.H., Hwang J. Susceptibility constants of Escherichia coli and Bacillus subtilis to silver and copper nanoparticles. Sci. Total Environ., 2007, 373, P. 572-575.

[15] Ruparelia J.P., Chatterjee A.K., Duttagupta S.P., Mukherji S. Strain specificity in antimicrobial activity of silver and copper nanoparticles. ActaBiomater., 2008, 4, P. 707-716.

[16] Hoseinzadeh E., Makhdoumi P., et al. A review on nano-antimicrobials: Metal nanoparticles, methods and mechanisms. Curr. Drug Metab., 2017, 18 (2), P. 120-128.

[17] Azam A, Ahmed A.S., et al. Antimicrobial activity of metal oxide nanoparticles against Gram-positive and Gram-negative bacteria: a comparative study. Int. J. Nanomed., 2012, 7, P. 6003-6009.

[18] Morones J.R., Elechiguerra J.L., et al. The bactericidal effect of silver nanoparticles. Nanotech., 2005, 16 (10), P. 2346-2353.

[19] Wahab R., Kim Y-S., et al. Formation of ZnO Micro-Flowers Prepared via Solution Process and their Antibacterial Activity. Nanoscale Res. Lett., 2010, 5, P. 1675-1681.

[20] Ahamed M., AlhadlaqHisham A., et al. Synthesis, characterization, and antimicrobial activity of copper oxide nanoparticles. J. Nanomater., 2014, 637858

[21] Shahzadi T., Zaib M., et al. Synthesis of Eco-friendly Cobalt Nanoparticles Using Celosia argentea Plant Extract and Their Efficacy Studies as Antioxidant, Antibacterial, Hemolytic and Catalytical Agent. Arab. J. Sci. Eng., 2019, 44, P. 6435-6444.

[22] Anwar A., Numan A., et al. Cobalt nanoparticles as novel nanotherapeutics against Acanthamoebacastellanii. Parasite. Vector, 2019, 12 (1), P. 280.

[23] Raza M.A., Kanwal Z., Riaz S., Naseem S. Synthesis, characterization and antibacterial properties of nano-sized cobalt particles. The 2016 World Congress on Advances in Civil, Environmental and Materials Research (ACEM'16), Jeju Island, Korea, 2016.

[24] Venkatesan K., Supriya R., et al. Cobalt ferrite $\left(\mathrm{CoFe}_{2} \mathrm{O}_{4}\right)$ nanoparticles for evaluation of antibacterial activity. J. Ind. Chem. Soc., 2015, 92, P. 637-639.

[25] Gingasu D., Mindru I., et al. Green synthesis methods of $\mathrm{CoFe}_{2} \mathrm{O}_{4}$ and Ag-CoFe $\mathrm{O}_{2} \mathrm{O}_{4}$ nanoparticles using hibiscus extracts and their antimicrobial potential. J. Nanomater., 2016, 2106756.

[26] Jung W.K., Koo H.C., et al. Antibacterial activity and mechanism of action of the silver ion in Staphylococcus aureus and Escherichia coli. Appl. Environ. Microbiol., 2008, 74 (7), P. 2171-2178.

[27] Li H., Chen Q., Zhao J., Urmila K. Enhancing the antimicrobial activity of natural extraction using the synthetic ultrasmall metal nanoparticles. Sci. Rep., 2015, 5, P. 11033.

[28] Armentano I., Arciola C.R., et al. The interaction of bacteria with engineered nanostructured polymeric materials: A review. Sci. World J., 2014, 2014, 410423.

[29] Gao W., Thamphiwatana S., Angsantikul P., Zhang L. Nanoparticle approaches against bacterial infections. Wiley Interdiscip. Rev. Nanomed. Nanobiotechnol., 2014, 6, P. 532-547.

[30] Luan B., Huynh T., Zhou R. Complete wetting of graphene by biological lipids. Nanoscale, 2016, 8, P. $5750-5754$.

[31] Xu Y., Wei M.T., et al. Exposure to $\mathrm{TiO}_{2}$ nanoparticles increases staphylococcus aureus infection of HeLa cells. J. Nanobiotechnol., 2016, 14, P. 34.

[32] Zhang W., Li Y., Niu J.F., Chen Y.S. Photogeneration of reactive oxygen species on uncoated silver, gold, nickel, and silicon nanoparticles and their antibacterial effects. Langmuir, 2013, 29 (15), P. 4647-4651.

[33] Pelgrift R.Y., Friedman A.J. Nanotechnology as a therapeutic tool to combat microbial resistance. Adv. Drug Deliv., 2013, 65, P. 1803-1815.

[34] Raffi M., Hussain F., et al. Antibacterial characterization of silver nanoparticles against E. coli ATCC-15224. J. Mater. Sci. Technol., 2008, 24, P. 192-196.

[35] Dizaj S.M., Lotfipour F., et al. Antimicrobial activity of the metals and metal oxide nanoparticles. Mater. Sci. Eng. C, 2014,44 , P. $278-284$.

[36] Ivask A., Kurvet I., et al. Size-dependent toxicity of silver nanoparticles to bacteria, yeast, algae, crustaceans and mammalian cells in vitro. PLOS One, 2014, 9 (7), e102108.

[37] You C., Han C., et al. The progress of silver nanoparticles in the antibacterial mechanism, clinical application and cytotoxicity. Mol. Biol. Rep., 2012, 39, P. 9193-9201.

[38] Mukha I.P., Eremenko A.M., et al. Antimicrobial activity of stable silver nanoparticles of a certain size. Appl. Biochem. Micro., 2013, 49 (2), P. 199-206.

[39] Selvarani M., Prema P. Evaluation of antibacterial efficacy of chemically synthesized copper and zerovalent iron nanoparticles. Asian J. Pharm. Clin. Res., 2013, 6, P. 223-227.

[40] Kim J.S., Kuk E., et al. Antimicrobial effects of silver nanoparticles. Nanomed., 2007, 3, P. 95-101.

[41] Sondi I., Salopek-Sondi B. Silver nanoparticles as antimicrobial agent: a case study on E. coli as a model for Gram-negative bacteria. $J$. Colloid. Interf. Sci., 2004, 275, P. 177-182.

[42] Deravi L.F., Swartz J.D., Wright D.W. The biomimetic synthesis of metal oxide nanomaterials, Wiley, 2010.

[43] Mu H., Tang J., et al. Potent antibacterial nanoparticles against biofilm and intracellular bacteria. Sci. Rep., 2016,6, P. 18877.

[44] Lai H-Z., Chen W-Y., Wu C-Y., Chen Y-C. Potent antibacterial nanoparticles for pathogenic bacteria. ACS Appl. Mater. Interfaces, 2015, 7, P. 2046-2054. 